\title{
Enantioselective Conjugate Addition of Catalytically Generated Zinc Homoenolate
}

\author{
Yoshiya Sekiguchi and Naohiko Yoshikai* \\ Division of Chemistry and Biological Chemistry, School of Physical and Mathematical Sciences, Nanyang Technological \\ University, Singapore 637371, Singapore
}

Supporting Information Placeholder

ABSTRACT: We report herein an enantioselective conjugate addition reaction of a zinc homoenolate, catalytically generated via ring opening of a cyclopropanol, to an $\alpha, \beta$-unsaturated ketone. The reaction is promoted by a zinc aminoalkoxide catalyst generated from $\mathrm{Et}_{2} \mathrm{Zn}$ and a chiral $\beta$-amino alcohol to afford 1,6diketones, which undergo, upon heating, intramolecular aldol condensation to furnish highly substituted cyclopentene derivatives with good to high enantioselectivities. The reaction has proved applicable to various 1-substituted cyclopropanols as well as chalcones and related enones. The chiral amino alcohol has proved to enable ligand-accelerated catalysis of the homoenolate generation and its conjugate addition. Positive nonlinear effects and lower reactivity of a racemic catalyst have been observed, which can be attributed to a stable and inactive heterochiral zinc aminoalkoxide dimer.

Metal homoenolates represent useful organometallic nucleophiles that allow for umpolung functionalization of the $\beta$ position of a carbonyl group. ${ }^{1}$ Pioneering studies by Nakamura and Kuwajima established 1-alkoxy-1siloxycyclopropanes as precursors to various main group and transition metal ester homoenolates, among which zinc homoenolate proved to be a particularly versatile nucleophile for fundamental $\mathrm{C}-\mathrm{C}$ bond forming reactions including 1,2/1,4-addition, allylic substitution, and crosscoupling (Scheme 1a). ${ }^{2}$ Nevertheless, an enantioselective variant of such transformations remains elusive. This is in sharp contrast to the fact that the catalytic enantioselective 1,2/1,4-addition and allylic substitution reactions of simple dialkylzincs have been developed and matured to the level that they now serve as benchmarks for the evaluation of new chiral ligands and catalysts. ${ }^{3}$ For example, more than 800 and 100 articles can be found for the enantioselective addition of $\mathrm{Et}_{2} \mathrm{Zn}$ to benzaldehyde and cyclohexenone, respectively, while no analogous asymmetric reaction involving a preformed zinc homoenolate has been reported. ${ }^{4}$

Recently, cyclopropanols have emerged as viable precursors for the in situ-generation of ketone homoenolates. ${ }^{1 \mathrm{c}-\mathrm{f}}$ One of seminal studies in this context is Cha's work on allylic and propargylic substitutions mediated by stoichiometric $\mathrm{Et}_{2} \mathrm{Zn}$ in combination with $\mathrm{Cu}(\mathrm{I})$ salt, where equilibrium generation of zinc homoenolate from the corresponding cyclopropoxide and its transmetalation to $\mathrm{Cu}(\mathrm{I})$ are likely involved (Scheme 1b). ${ }^{5}$ However, such in situ-generated zinc homoenolate has also not been exploited for an enantioselective $\mathrm{C}-\mathrm{C}$ bond formation. ${ }^{6,7}$

Herein, we report on an enantioselective conjugate addition (ECA) of a catalytically generated chiral zinc homoenolate to $\alpha, \beta$-unsaturated ketones (Scheme 1c). ${ }^{8,9}$ A catalyst generated from $\mathrm{Et}_{2} \mathrm{Zn}$ and a chiral $\beta$-amino alcohol promotes ring-opening addition of cyclopropanols to chalcone and related enones, which displays the feature of ligand-accelerated catalysis and represents a rare example of transition metal-free ECA of organozinc reagents. ${ }^{10}$ Upon heating, the 1,6-diketone products undergo facile intramolecular aldol condensation to afford multisubstituted cyclopentene derivatives with high enantioselectivity. ${ }^{11}$

Scheme 1. Generation and $\mathrm{C}-\mathrm{C}$ Bond-Forming Reactions of Zinc Homoenolate 
(a) Preformed zinc homoenolate (Nakamura/Kuwajima)

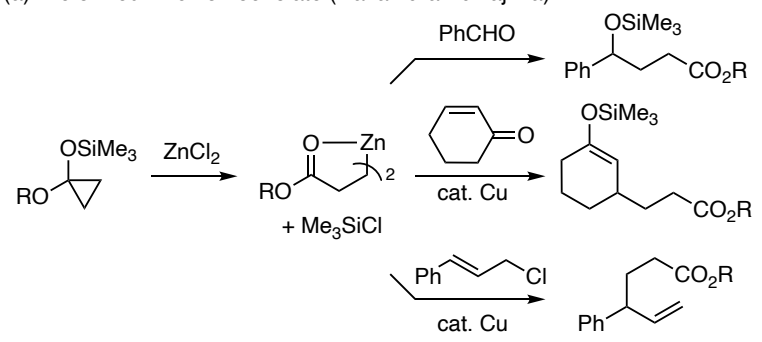

(b) In situ-generated zinc homoenolate (Cha)

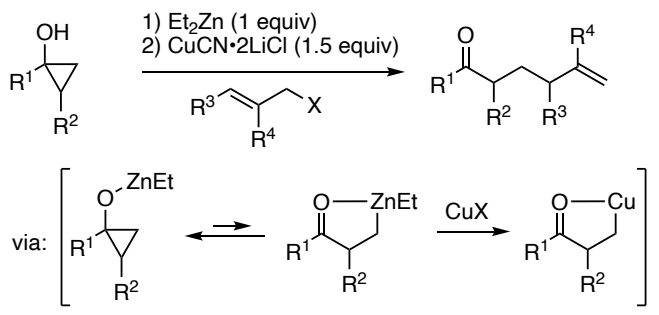

(c) This work: Catalytically generated chiral zinc homoenolate

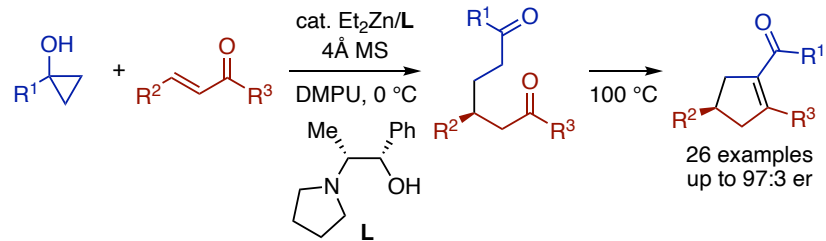

- Ligand-accelerated homoenolate generation and ECA

- Transition metal-free organozinc ECA

- Enantioenriched cyclopentane scaffolds

In light of Cha's seminal work on the equilibrium generation of zinc homoenolate from zinc cyclopropoxide, ${ }^{5}$ our first question was whether it is possible to exploit such process for $\mathrm{C}-\mathrm{C}$ bond formation catalytic in zinc. Thus, we initially examined the reaction between 1-phenylcyclopropanol (1a) and chalcone (2a). Upon brief screening, a catalytic system comprised of $\mathrm{Et}_{2} \mathrm{Zn}(10 \mathrm{~mol} \%)$ and $\mathrm{DABCO}(10 \mathrm{~mol} \%)$ was found to promote the desired ring-opening conjugate addition in DMSO at $80^{\circ} \mathrm{C}$, affording 1,6-diketone 3aa in $47 \%$ yield along with a small amount of cyclopentene derivative 4 aa as a result of intramolecular aldol condensation of $\mathbf{3 a a}$ (eq 1). By adding molecular sieves $4 \AA$, the reaction gave $4 \mathbf{a a}$ as the dominant product.

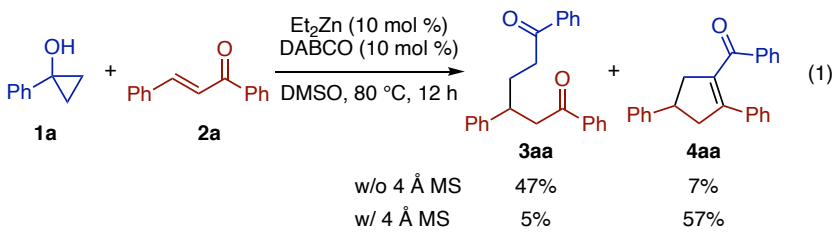

Given the feasibility of zinc-catalyzed generation and conjugate addition of homoenolate from cyclopropanol, we next attempted on rendering the reaction enantioselective. In this respect, we explored a host of chiral $\beta$-amino alcohols as ligands, which have found extensive use in the enantioselective addition of dialkylzincs $^{3 \mathrm{a}}$ as well as preformed or in situ-generated zinc acetylide. ${ }^{12}$ Upon screening of ligands, solvents, and other reaction conditions, a few amino alcohols (L1, L3, and L5-L7) emerged as promising ligands (Table 1, entries 1-7; see also Tables S1 and S2). For example, the reaction of $\mathbf{1 a}$ and $\mathbf{2 a}$ in the presence of $\mathrm{Et}_{2} \mathrm{Zn}$ and the norephedrine derivative L6 (10 mol \% each) and $4 \AA$ MS in DMSO was performed at $30^{\circ} \mathrm{C}$ for $24 \mathrm{~h}$, followed by heating at $100{ }^{\circ} \mathrm{C}$ for $6 \mathrm{~h}$, to afford the cyclopentene $4 \mathrm{aa}$ in $73 \%$ yield with $87: 13$ er (entry 6 ). The use of DMPU as the solvent improved the enantioselectivity to $93: 7 \mathrm{er}$ (entry 8). By performing the ECA step at $0{ }^{\circ} \mathrm{C}$ for $48 \mathrm{~h}$ with an increased catalyst loading (15 mol \%), an additional improvement in the yield $(89 \%)$ and the enantioselectivity (95:5 er) was achieved (entry 9).

Table 1. Zinc-Catalyzed Addition of 1-Phenylcyclopropanol (1a) to Chalcone (2a) ${ }^{a}$

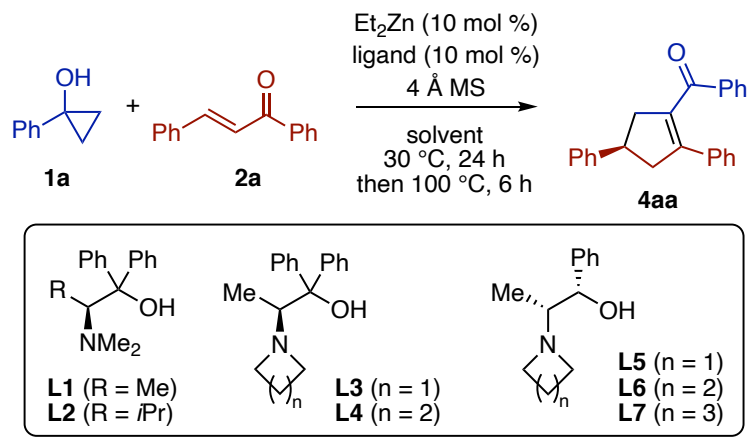

$\begin{array}{lllll}\text { entry } & \text { ligand } & \text { solvent } & \text { yield }(\%)^{b} & \text { er }^{c} \\ 1 & \text { L1 } & \text { DMSO } & 88 & 82: 18 \\ 2 & \text { L2 } & \text { DMSO } & 65 & 47: 54 \\ 3 & \text { L3 } & \text { DMSO } & 69 & 79: 21 \\ 4 & \text { L4 } & \text { DMSO } & 63 & 52: 48 \\ 5 & \text { L5 } & \text { DMSO } & 71 & 77: 23 \\ 6 & \text { L6 } & \text { DMSO } & 73 & 87: 13 \\ 7 & \text { L7 } & \text { DMSO } & 50 & 82: 18 \\ 8 & \text { L6 } & \text { DMPU } & 79 & 93: 7 \\ 9^{d} & \text { L6 } & \text { DMPU } & 89 & 95: 5\end{array}$

${ }^{a}$ The reaction was performed using $0.15 \mathrm{mmol}$ of $\mathbf{1 a}$ and $0.1 \mathrm{mmol}$ of $2 \mathrm{a}$ in $0.3 \mathrm{~mL}$ solvent $(0.33 \mathrm{M}){ }^{b}$ Determined by GC using mesitylene as an internal standard. ${ }^{c}$ Determined by chiral HPLC. ${ }^{d} 15 \mathrm{~mol} \%$ each of $\mathrm{Et}_{2} \mathrm{Zn}$ and $\mathbf{L 6}$ was used. The reaction was performed at $0{ }^{\circ} \mathrm{C}$ for $48 \mathrm{~h}$ and then at $100{ }^{\circ} \mathrm{C}$ for $6 \mathrm{~h}$.

With the optimized catalytic system (Table 1, entry 9) in hand, we explored the scope of the present homoenolate ECA. First, a variety of cyclopropanols were subjected to the reaction with chalcone (2a) (Scheme 2). Various 1-arylcyclopropanols participated in the ring-opening conjugate addition/intramolecular aldol cascade, thus affording cyclopentenes 4aa-4ha in moderate to high yields and enantioselectivities with tolerance to methoxy, bromo, iodo, and trifluoromethyl 
groups. A modest trend of decreasing enantioselectivity with electron-withdrawing aryl group was observed, as manifested in the case of para-trifluoromethyl group (see 4ea). The absolute configuration of the Br-substituted product 4da was determined to be $(R)$ by X-ray crystallographic analysis. The reaction of 1a could be performed on a $3 \mathrm{mmol}$ scale without decrease in the enantioselectivity. Importantly, the reaction of 1a and $\mathbf{2 a}$, when quenched before the aldol step, gave the 1,6diketone product 3aa in good yield with enantioselectivity (95:5 er) identical to that of 4aa. 1-(2Thienyl)cyclopropanol also smoothly took part in the reaction to furnish the product 4ia with $96: 4$ er. The reactions of 1-alkylcyclopropanols were somewhat sluggish and required higher temperature. Nonetheless, the corresponding cyclopentene products (4ja and $\mathbf{4 k a})$ were obtained with excellent enantioselectivity (97:3 er). A racemic bicyclic cyclopropanol 11 (2 equiv) underwent selective cleavage of the less substituted $\mathrm{C}-\mathrm{C}$ bond, and afforded the desired conjugate adduct 3la as a diastereomer mixture (78:22 dr) in 96\% yield, with $98: 2$ and 90:10 ers for the major and minor diastereomers, respectively. Meanwhile, the unreacted $\mathbf{1 l}$ was isolated in the form of benzoyl ester in $24 \%$ yield (based on 11) with 96:4 er. This outcome can be rationalized by highly selective kinetic resolution in the ring-opening step and moderately enantioselective conjugate addition of each diastereomeric zinc homoenolate (see Scheme S1).

Scheme 2. Asymmetric Ring-Opening Addition of Various Cyclopropanols to 2a ${ }^{a}$

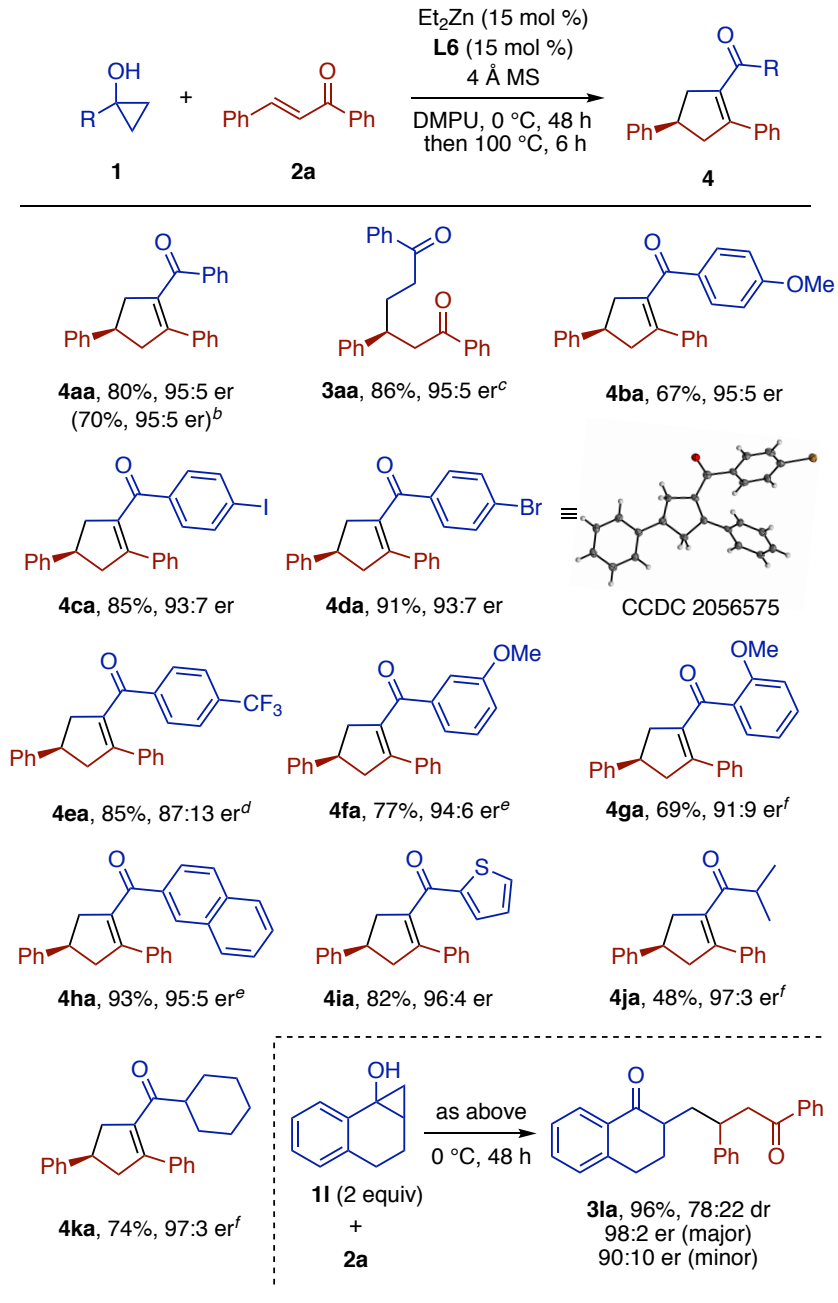

${ }^{a}$ The reaction was performed on a $0.3 \mathrm{mmol}$ scale under the conditions in Table 1 , entry $9 .{ }^{b} 3$ mmol-scale reaction. ${ }^{c}$ The reaction was quenched without aldol condensation step. ${ }^{d}$ The ECA step was performed at $30^{\circ} \mathrm{C}$ for $12 \mathrm{~h} .{ }^{e}$ The ECA step was performed at $0{ }^{\circ} \mathrm{C}$ for $72 \mathrm{~h} .{ }^{f}$ The ECA step was performed at $30^{\circ} \mathrm{C}$ for $48 \mathrm{~h}$.

We next explored the addition of $\mathbf{1 a}$ to various $\alpha, \beta-$ unsaturated ketones (Scheme 3). Chalcone-type di(hetero)aryl-substituted enones proved to be excellent substrates, affording the corresponding cyclopentenes 4ab-4aj in moderate to high yields with 92:8 to $96: 4$ er. A variety of functionalized aryl and heteroaryl groups could be tolerated on both ends of the enone skeleton. An $\alpha, \beta, \gamma, \delta$-unsaturated ketone underwent selective 1,4addition of the homoenolate to give the alkenylsubstituted cyclopentene 4ak with 97:3 er. Meanwhile, enones bearing an alkyl substituent at either the $\beta$ position or the acyl position failed to participate in the reaction under the standard conditions. Using $\mathbf{L 3}$ as the ligand, such substrates afforded the desired products 4al and 4am with moderate enantioselectivities (ca. 80:20 er). $\beta$ Trifluoromethyl enone also took part in the reaction, albeit in modest yield and enantioselectivity (see 4an). The reaction of (E)-2-benzylidenecyclohexan-1-one furnished, after $3 \mathrm{~h}$ of the aldol condensation step, a 
bicyclic product 4ao as a mixture of diastereomers (80:20 dr) with ers of 84:16 (major) and 70:30 (minor). The relative stereochemistry of the major diastereomer of $\mathbf{4 a o}$ was confirmed by X-ray crystallographic analysis of its anilide derivative 5ao. Notably, extension of the condensation step for this product resulted in transposition of the $\mathrm{C}=\mathrm{C}$ bond to the bicyclic juncture to give a tetrasubstituted olefin isomer (66\% yield, 50:50 dr and 80:20 er for both diastereomers), presumably via zinc dienolate intermediate (see Scheme S2). Note that the present catalytic system failed to engage cyclic enones such as cyclohexanone as the reaction partner for $\mathbf{1 a}$.

\section{Scheme 3. Asymmetric Ring-Opening Addition of 1a} to Various Enones ${ }^{a}$

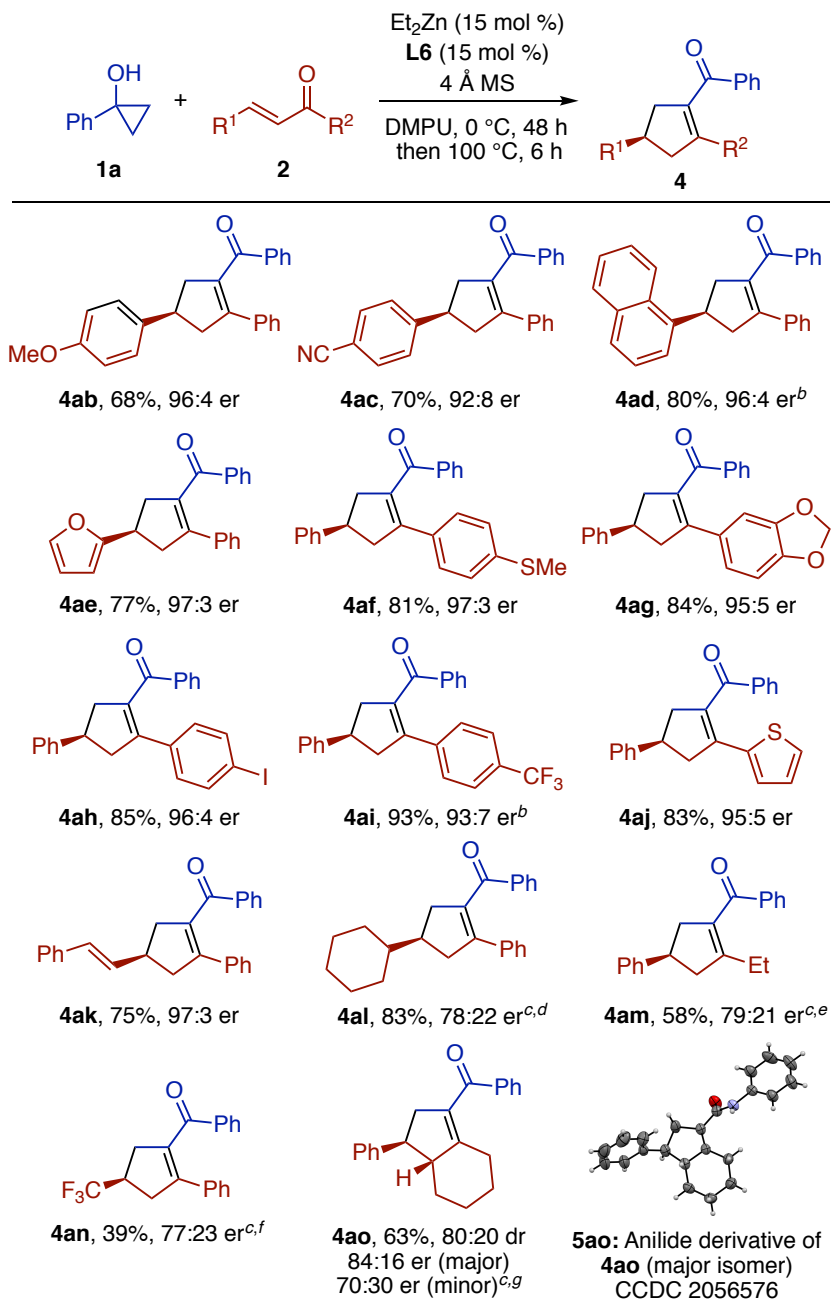

${ }^{a}$ The reaction was performed on a $0.3 \mathrm{mmol}$ scale under the conditions in Table 1 , entry $9 .{ }^{b}$ The aldol condensation step was performed at $50{ }^{\circ} \mathrm{C}$ for $16 \mathrm{~h} .{ }^{c} \mathbf{L} 3$ was used instead of L6. ${ }^{d}$ The reaction was performed in DMSO at $40{ }^{\circ} \mathrm{C}$ for $48 \mathrm{~h}$ and then at $100{ }^{\circ} \mathrm{C}$ for $6 \mathrm{~h}$. ${ }^{e}$ The ECA step was performed at $30{ }^{\circ} \mathrm{C}$ for $48 \mathrm{~h}$. ${ }^{f}$ The ECA step was performed at $30{ }^{\circ} \mathrm{C}$ for $24 \mathrm{~h}$. ${ }^{g}$ The aldol condensation step was performed for $3 \mathrm{~h}$.

To gain insight into the ligand effect and the zinc species involved in the present homoenolate ECA, we

performed a series of mechanistic experiments. The model reaction of 1a and 2a (performed at $30{ }^{\circ} \mathrm{C}$ to facilitate the reaction at low catalyst loadings and/or with low-ee catalysts) was found to display a concentrationdependent nonlinear effects (NLEs; Figure 1a). ${ }^{13}$ Thus, a clear positive NLE was observed with $20 \mathrm{~mol} \%$ catalyst loading (67 mM [Zn]), while NLE became rather weak upon reducing the catalyst loading down to $10 \mathrm{~mol} \%$ (33 $\mathrm{mM}[\mathrm{Zn}])$. Note that the reaction temperature $\left(30^{\circ} \mathrm{C}\right.$ vs 0 ${ }^{\circ} \mathrm{C}$ ) and the solvent composition (DMPU vs DMPU/THF) had only minor impact on NLE (see Figures S5 and S6). The ee of $\mathbf{L 6}$ was also found to significantly influence the reaction rate. Thus, the initial rate of the reaction using enantiopure L6 was found to be more than four times faster than that using racemic L6 (Figure 1b). These observations suggest the role of homochiral and heterochiral $\mathrm{Zn}$ aminoalkoxide dimers as off-cycle species and the reluctance of the latter to dissociate into catalytically active monomeric $\mathrm{Zn}$ species (vide infra). ${ }^{13 \mathrm{~b}}$ The ability of the amino alcohol to accelerate the whole reaction process as well as the ring opening step alone was clearly demonstrated by control experiments. Thus, no reaction took place between $1 \mathbf{a}$ and $\mathbf{2 a}$ by omitting $\mathbf{L 6}$ from or replacing $\mathbf{L 6}$ with DABCO in the standard conditions. The reaction of $1 \mathrm{a}$ alone under the standard conditions quantitatively produced propiophenone 1a' after $48 \mathrm{~h}$, while the ring opening was rather sluggish by omitting L6 or using DABCO (Figure 1c). Lastly, we probed the solvent effect (DMPU, THF, and their mixed solvents) on the model reaction (Figure 1d). The enantioselectivity proved rather insensitive to the solvent composition. By contrast, the product yield dropped significantly in pure THF (15\%) owing to competitive Michael addition of propiophenone enolate to $\mathbf{2 a}$, while the yield, albeit with some fluctuation, remained substantial $(\geq 50 \%)$ in the mixed solvents (\%THF up to $75 \%$ ).

(a)

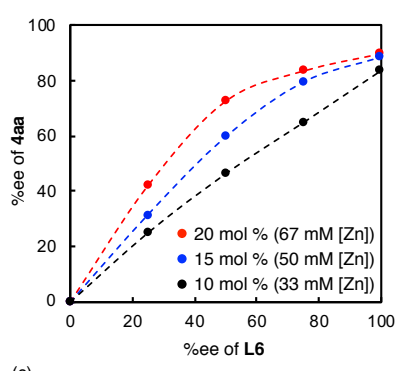

(c)

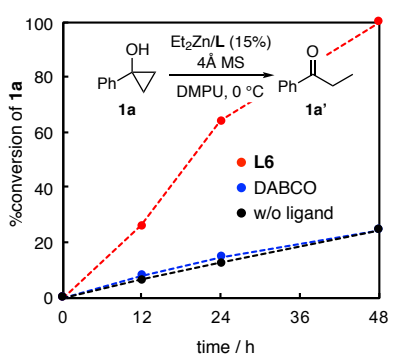

(b)

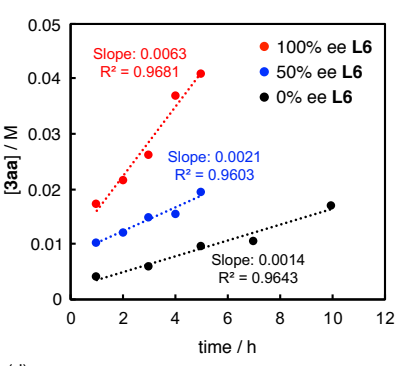

(d)

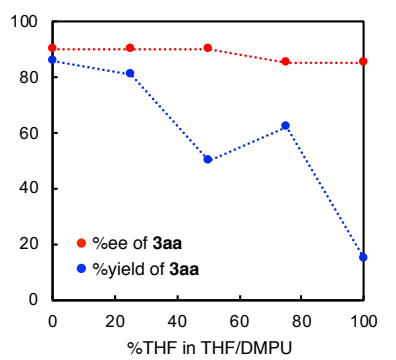


Figure 1. Mechanistic experiments. (a) Nonlinear effects with different catalyst loadings of $\mathrm{Et}_{2} \mathrm{Zn} / \mathbf{L 6}\left(30^{\circ} \mathrm{C}, 30 \mathrm{~h}\right.$ and $100{ }^{\circ} \mathrm{C}, 6 \mathrm{~h}$ ). (b) Initial reaction progress using $\mathbf{L 6}$ of different ees ( $15 \mathrm{~mol} \%$ catalyst loading, $\left.0{ }^{\circ} \mathrm{C}\right)$. (c) Ringopening of 1a alone. (d) Ees and yields of 3aa in THF/DMPU mixed solvents.

On the basis of the above experiments and the literature knowledge on chiral amino alcohol-catalyzed organozinc addition, ${ }^{3 a, 13 b}$ we propose a catalytic cycle involving a mixed zinc alkoxide $\mathbf{A}$ derived from $\mathrm{Et}_{2} \mathrm{Zn}$, the amino alcohol, and the cyclopropanol $\mathbf{1}$ as well as its homochiral and heterochiral dimers ([A $]_{2}$ and $[\mathbf{A} \bullet$ ent-A $]$; Scheme 4). The monomeric mixed alkoxide $\mathbf{A}$ would act as a catalytically competent species, undergoing ring-opening to generate the corresponding homoenolate B. Upon coordination of the enone $\mathbf{2}$, the homoenolate $\mathbf{B}$ would undergo asymmetric conjugate addition to give a zinc enolate $\mathbf{D}$, which would deprotonate $\mathbf{1}$ to give the 1,6diketone while regenerating the mixed alkoxide $\mathbf{A}$. When there is no enone or ECA is sluggish, the homoenolate $\mathbf{B}$ can be protonated by $\mathbf{1}$ to give the corresponding ketone 1', which can further participate in enolate Michael addition under zinc catalysis. Based on the solvent effect (Figure 1d), the ability of DMPU as a Lewis base to Zn, rather than its bulk polarity as a solvent, appears to be critical for the preferential CA of the homoenolate over its protonation.

\section{Scheme 4. Possible Catalytic Cycle}

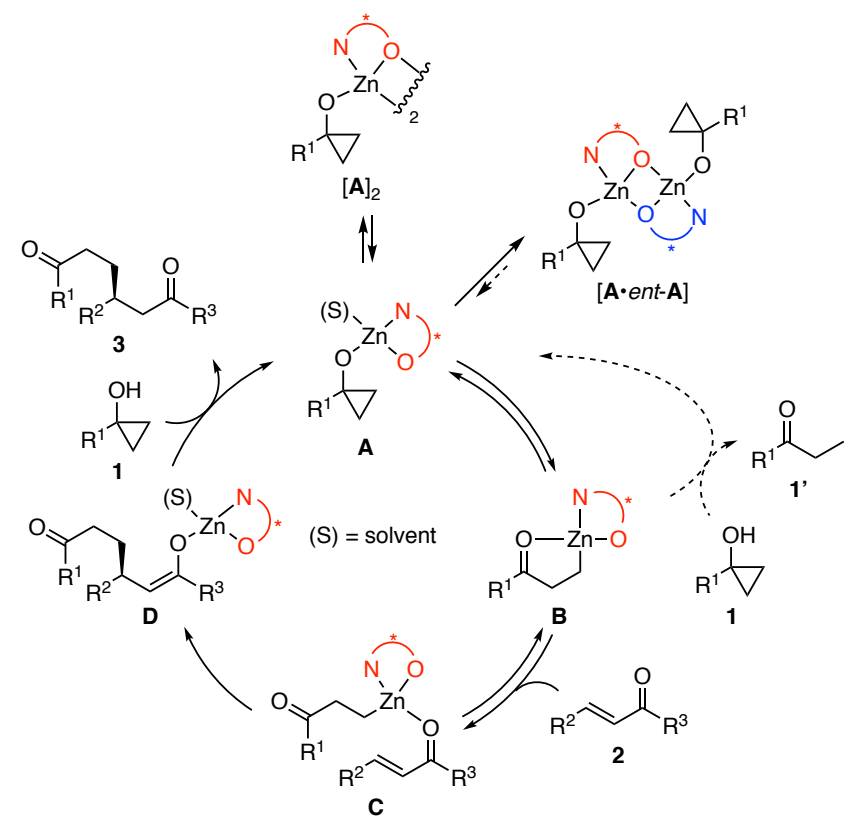

Selected transformations of the enantioenriched cyclopentene product 4aa are shown in Scheme 5. Conversion of the benzoyl group to an anilide moiety via Beckmann rearrangement was achieved through oxime formation and subsequent treatment with $\mathrm{Tf}_{2} \mathrm{O}$, affording the product 5 in good yield. Epoxidation with $m \mathrm{CPBA}$ provided the tetrasubstituted epoxide $\mathbf{6}$ in a diastereoselective manner, albeit in a modest yield.
Generation of a dienolate species from 4aa and LiHMDS was followed by trapping with benzyl bromide, which took place regioselectively at the $\alpha$ position to generate a quaternary stereocenter in the product 7 in a diastereoselective manner.

\section{Scheme 5. Product Transformations}

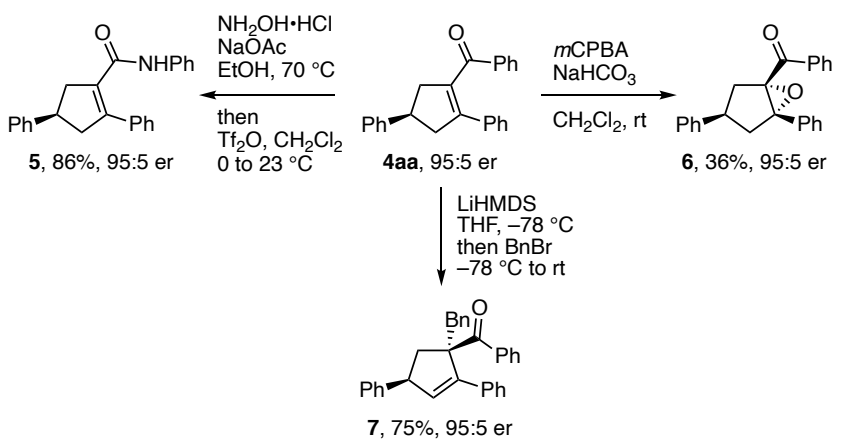

In summary, we have developed an enantioselective ring-opening conjugate addition of cyclopropanols to $\alpha, \beta$ unsaturated ketones via catalytic generation of zinc homoenolate. A chiral $\beta$-amino alkoxide ligand on zinc accelerates the ring-opening of a cyclopropanol and aids in enantioselective addition of the resulting homoenolate to chalcones and related enones without assistance of transition metal catalyst. The ensuing intramolecular aldol condensation of the 1,6-diketone products allow for facile preparation of multisubstituted cyclopentene derivatives with good to high enantioselectivity. The reaction represents the first example of enantioselective transformation of zinc homoenolate, and also a rare example of asymmetric transformations of metal homoenolates in general. Further development and mechanistic study of synthetic transformations involving catalytically generated metal homoenolates are ongoing in our laboratory.

\section{ASSOCIATED CONTENT}

Experimental procedures and characterization data for all the new products (PDF).

Crystallographic data for $\mathbf{4 d a}$ (CIF)

Crystallographic data for $\mathbf{5 a o}$ (CIF)

\section{AUTHOR INFORMATION}

\section{Corresponding Author}

*nyoshikai@ntu.edu.sg

\section{Notes}

The authors declare no competing financial interests.

\section{ACKNOWLEDGMENT}

This work was supported by the Ministry of Education (Singapore) and Nanyang Technological University (MOE2016-T2-2-043 and RG101/19). We thank Dr. 
Yongxin Li (Nanyang Technological University) for his assistance with the X-ray crystallographic analysis.

\section{REFERENCES}

(1) (a) Kuwajima, I.; Nakamura, E. Metal Homoenolates from Siloxycyclopropanes. Top. Curr. Chem. 1990, 155, 1-39. (b) Kimura, M. In Comprehensive Organic Synthesis II; Knochel, P., Ed.; Elsevier: 2014; Vol. 2. (c) Mills, L. R.; Rousseaux, S. A. L. Modern Developments in the Chemistry of Homoenolates. Eur. J. Org. Chem. 2019, 8-26. (d) Nikolaev, A.; Orellana, A. Transition-Metal-Catalyzed $\mathrm{C}-\mathrm{C}$ and $\mathrm{C}-\mathrm{X}$ Bond-Forming Reactions Using Cyclopropanols. Synthesis 2016, 48, 1741-1768. (e) McDonald, T. R.; Mills, L. R.; West, M. S.; Rousseaux, S. A. L. Selective Carbon-Carbon Bond Cleavage of Cyclopropanols. Chem. Rev. 2021, 121, 3-79. (f) Sekiguchi, Y.; Yoshikai, N. Metal-Catalyzed Transformations of Cyclopropanols via Homoenolates. Bull. Chem. Soc. Jpn. 2021, 94, 265-280.

(2) (a) Nakamura, E.; Kuwajima, I. Copper-Catalyzed Acylation and Conjugate Addition of Zinc Homoenolate. Synthesis of 4- and 5-Oxo Esters. J. Am. Chem. Soc. 1984, 106, 3368-3370. (b) Nakamura, E.; Aoki, S.; Sekiya, K.; Oshino, H.; Kuwajima, I. Carbon-Carbon BondForming Reactions of Zinc Homoenolate of Esters. A Novel ThreeCarbon Nucleophile with General Synthetic Utility. J. Am. Chem. Soc. 1987, 109, 8056-8066.

(3) (a) Soai, K.; Niwa, S. Enantioselective Addition of Organozinc Reagents to Aldehydes. Chem. Rev. 1992, 92, 833-856. (b) Yamada, K.; Tomioka, K. Copper-Catalyzed Asymmetric Alkylation of Imines with Dialkylzinc and Related Reactions. Chem. Rev. 2008, 108, 2874 2886. (c) Kobayashi, S.; Mori, Y.; Fossey, J. S.; Salter, M. M. Catalytic Enantioselective Formation of C-C Bonds by Addition to Imines and Hydrazones: A Ten-Year Update. Chem. Rev. 2011, 111, 2626-2704. (d) Alexakis, A.; Backvall, J. E.; Krause, N.; Pamies, O.; Dieguez, M. Enantioselective Copper-Catalyzed Conjugate Addition and Allylic Substitution Reactions. Chem. Rev. 2008, 108, 2796-2823. (e) Jerphagnon, T.; Pizzuti, M. G.; Minnaard, A. J.; Feringa, B. L. Recent Advances in Enantioselective Copper-Catalyzed 1,4-Addition. Chem. Soc. Rev. 2009, 38, 1039-1075.

(4) According to Reaxys search (accessed on January 15, 2021).

(5) Das, P. P.; Belmore, K.; Cha, J. K. $\mathrm{S}_{\mathrm{N}} 2$ ' Alkylation of Cyclopropanols via Homoenolates. Angew. Chem. Int. Ed. 2012, 51, 9517-9520.

(6) (a) Parida, B. B.; Das, P. P.; Niocel, M.; Cha, J. K. C-Acylation of Cyclopropanols: Preparation of Functionalized 1,4-Diketones. Org. Lett. 2013, 15, 1780-1783. (b) Murali, R. V. N. S.; Rao, N. N.; Cha, J. K. C-Alkynylation of Cyclopropanols. Org. Lett. 2015, 17, 3854-3856. (c) Mills, L. R.; Barrera Arbelaez, L. M.; Rousseaux, S. A. L. Electrophilic Zinc Homoenolates: Synthesis of Cyclopropylamines from Cyclopropanols and Amines. J. Am. Chem. Soc. 2017, 139, $11357-11360$

(7) (a) Nomura, K.; Matsubara, S. Preparation of Zinc-Homoenolate from $\alpha$-Sulfonyloxy Ketone and Bis(Iodozincio)Methane. Chem. Lett. 2007, 36, 164-165. (b) Nomura, K.; Matsubara, S. Stereospecific Construction of Chiral Tertiary and Quaternary Carbon by Nucleophilic Cyclopropanation with Bis(Iodozincio)Methane. Chem. Asian J. 2010, 5, 147-152.

(8) In fact, examples of enantioselective transformation of catalytically generated metal homoenolates remain very rare. See the following references and ref 9: (a) Yang, J.; Shen, Y.; Lim, Y. J.; Yoshikai, N. Divergent Ring-Opening Coupling between Cyclopropanols and Alkynes under Cobalt Catalysis. Chem. Sci. 2018, 9, 6928-6934. (b) Yang, J.; Sekiguchi, Y.; Yoshikai, N. CobaltCatalyzed Enantioselective and Chemodivergent Addition of Cyclopropanols to Oxabicyclic Alkenes. ACS Catal. 2019, 9, 56385644. (c) Huang, W.; Meng, F. Cobalt-Catalyzed Diastereo- and Enantioselective Hydroalkylation of Cyclopropenes with Cobalt Homoenolates. Angew. Chem. Int. Ed. 2020. DOI: 10.1002/anie.202012122.
(9) Burke, E. D.; Lim, N. K.; Gleason, J. L. Catalytic Enantioselective Homoaldol Reactions Using Binol Titanium(IV) Fluoride Catalysts. Synlett 2003, 390-392.

(10) Bräse, S.; Höfener, S. Asymmetric Conjugate Addition of Organozinc Compounds to $\alpha, \beta$-Unsaturated Aldehydes and Ketones with [2.2]Paracyclophaneketimine Ligands without Added Copper Salts. Angew. Chem. Int. Ed. 2005, 44, 7879-7881.

(11) For the importance of cyclopentanes in bioactive natural products and pharmacetutical drugs, see: (a) Hudlicky, T.; Price, J. D. Anionic Approaches to the Construction of Cyclopentanoids. Chem. Rev. 1989, 89, 1467-1486. (b) Das, S.; Chandrasekhar, S.; Yadav, J. S.; Gree, R. Recent Developments in the Synthesis of Prostaglandins and Analogues. Chem. Rev. 2007, 107, 3286-3337. (c) Liu, G.; Shirley, M. E.; Van, K. N.; McFarlin, R. L.; Romo, D. Rapid Assembly of Complex Cyclopentanes Employing Chiral, $\alpha, \beta$-Unsaturated Acylammonium Intermediates. Nat Chem 2013, 5, 1049-1057. (d) Heasley, B. Recent Developments in the Stereocontrolled Synthesis of Highly Substituted Cyclopentane Core Structures: From Drug Discovery Research to Natural Product Synthesis. Curr. Org. Chem. 2014, 18, 641-686.

(12) (a) Frantz, D. E.; Fassler, R.; Tomooka, C. S.; Carreira, E. M. The Discovery of Novel Reactivity in the Development of C-C BondForming Reactions: In Situ Generation of Zinc Acetylides with $\mathrm{Zn}(\mathrm{II}) / \mathrm{R}_{3} \mathrm{~N}$. Acc. Chem. Res. 2000, 33, 373-381. (b) Trost, B. M.; Weiss, A. H. The Enantioselective Addition of Alkyne Nucleophiles to Carbonyl Groups. Adv Synth Catal 2009, 351.

(13) (a) Satyanarayana, T.; Abraham, S.; Kagan, H. B. Nonlinear Effects in Asymmetric Catalysis. Angew. Chem. Int. Ed. 2009, 48, 456494. (b) Noyori, R.; Suga, S.; Oka, H.; Kitamura, M. Self and Nonself Recognition of Chiral Catalysts: The Origin of Nonlinear Effects in the Amino-Alcohol Catalyzed Asymmetric Addition of Diorganozincs to Aldehydes. Chem. Rec. 2001, 1, 85-100. (c) Girard, C.; Kagan, H. B. Nonlinear Effects in Asymmetric Synthesis and Stereoselective Reactions: Ten Years of Investigation. Angew. Chem. Int. Ed. 1998, 37, 2922-2959. (d) Noyori, R.; Kitamura, M. Enantioselective Addition of Organometallic Reagents to Carbonyl Compounds: Chirality Transfer, Multiplication, and Amplification. Angew. Chem. Int. Ed. 1991, 30, 4969. 


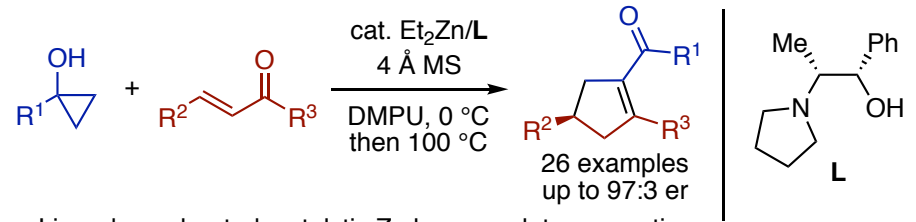

- Ligand-accelerated, catalytic Zn homoenolate generation

- TM-free enantioselective organozinc conjugate addition

- Enantioenriched cyclopentane scaffolds 